\title{
Análisis espacial de las condiciones de vulnerabilidad social, económica, física y ambiental en el territorio colombiano
}

\author{
SPATIAL ANALYSIS OF THE CONDITIONS OF SOCIAL, ECONOMIC, \\ PHYSICAL AND ENVIRONMENTAL VULNERABILITY IN THE \\ COLOMBIAN TERRITORY
}

ANALISE ESPACIAL DAS CONDIÇÕES DE VULNERABILIDADE SOCIAL, ECONÔMICA, FÍSICA E AMBIENTAL NO TERRITÓRIO COLOMBIANO

Carlos Alberto Durán Gil ${ }^{1}$

Para citar este artículo: Durán Gil, C. A. (2017). Análisis espacial de las condiciones de vulnerabilidad social, económica, física y ambiental en el territorio colombiano. Perspectiva Geográfica, 22(1), 11-32. doi: 10.19053/01233769.5956

Recepción:

27 de marzo de 2017

Evaluación:

16 de junio de 2017

Aprobación:

22 de junio de 2017

\section{Resumen}

Este artículo describe el desarrollo de una metodología generada desde el análisis espacial que permite determinar, cuantificar y categorizar la vulnerabilidad en sus dimensiones social, económica, física y ambiental, y caracterizar la suma de estas como vulnerabilidad total. Con base en información oficial desagregada a nivel municipal, se realiza una selección de factores incidentes en cada dimensión de la vulnerabilidad, los cuales son sujetos a un análisis exploratorio espacial para determinar comportamientos particulares de tipo asociativo. Estos factores, al ser adicionados de manera equiponderada a través de valores normalizados, permiten definir índices de vulnerabilidad, cuyos resultados se visualizan por medio de su representación cartográfica temática sobre el espacio colombiano, reflejando tendencias y patrones diferenciados, tanto de alta como de baja vulnerabilidad. Este enfoque permite com-

1 Ingeniero catastral y geodesta de la Universidad Distrital Francisco José de Caldas. Especialista en Análisis Espacial de la Universidad Nacional de Colombia. Profesional especializado del Grupo de Investigación y Desarrollo de la Dirección de Geoestadística del Departamento Administrativo Nacional de Estadística (DANE), donde lidera procesos relacionados con cartografía temática, análisis y modelado espacial. cdurangil@gmail.com 
prender la vulnerabilidad como un fenómeno multicausal y de connotación espacial, siendo esta una cuestión relevante para la evaluación focalizada de su impacto y la toma adecuada de decisiones.

Palabras clave: análisis espacial, análisis exploratorio de datos espaciales, autocorrelación espacial, patrones, vulnerabilidad.

\section{Abstract}

This article describes the development of a methodology generated from the spatial analysis that allows to determine, quantify and categorize vulnerability in its social, economic, physical and environmental dimensions, and characterize the sum of these as the total vulnerability. Based on official information disaggregated at the municipal level, a selection of incident factors in each vulnerability dimension is made, which are subject to an exploratory spatial analysis to determine particular behaviors of associative type. These factors, when added in an equal-weighted way through normalized values, allow defining vulnerability indexes, whose results are visualized through thematic cartographic representation on the Colombian space, reflecting different trends and patterns, both high and low vulnerability. This approach allows the understanding of vulnerability as a multi-causal and spatial connotation phenomenon, which is a relevant issue for the focused assessment of its impact and appropriate decision making.

Keywords: spatial analysis, exploratory analysis of spatial data, spatial autocorrelation, patterns, vulnerability.

\section{Resumo}

Este artigo descreve o desenvolvimento de uma metodologia gerada a partir da análise espacial que permite determinar, quantificar e categorizar a vulnerabilidade em suas dimensões social, econômica, física e ambiental, e caracterizar a soma destas como vulnerabilidade total. Com base em informação oficial desagregada a nível municipal, se realiza uma seleção de fatores incidentes em cada dimensão da vulnerabilidade, os quais são sujeitos a uma análise exploratória espacial para determinar comportamentos particulares do tipo associativo. Estes fatores, ao ser adicionados de maneira equiponderada através de valores normalizados, permitem definir índices de vulnerabilidade, cujos os resultados se visualizam por meio de sua representação cartográfica 
temática sobre o espaço colombiano, refletindo tendências e padrões diferenciados, tanta da alta como de baixa vulnerabilidade. Este enfoque permite compreender a vulnerabilidade como um fenômeno multicausal e de conotação espacial, sendo esta uma questão relevante para a evolução focalizada de seu impacto e a tomada adequada de decisões.

Palavra-chave: análise espacial, análise explorando os dados espaciais, autocorrelação espacial, padrões e vulnerabilidade. 


\section{Introducción}

Generalmente los análisis relacionados con la vulnerabilidad se abordan de manera diferencial, casi dicotómica, ya sea desde una perspectiva socioeconómica ligada a la población, los hogares y las viviendas, o desde un contexto eminentemente ambiental, relacionado exclusivamente a las características propias del medio físico. Sin embargo, las fragilidades territoriales se deben abordar desde una perspectiva geográfica, es decir, la relación del ser humano (sociedad) y el medio (naturaleza) en el que habita, con sus influencias recíprocas.

Ya que Colombia, sus departamentos y sus municipios son territorios de gran diversidad y complejidad, tanto en el contexto social de sus habitantes como en las características propias de su entorno físico, se hace indispensable establecer y analizar los factores asociados a las posibles vulnerabilidades generadas por estas condiciones territoriales de manera conjunta, las cuales pueden acentuar o mitigar el impacto de una posible amenaza e influir tanto en la resiliencia como en la adaptación posterior al perjuicio. Ante esto, se plantean los siguientes interrogantes que enmarcan el problema a resolver: ¿en el territorio colombiano la vulnerabilidad, en sus diferentes dimensiones, presenta patrones o tendencias particulares de tipo espacial? ¿Existen zonas que presentan de manera conjunta fragilidades sociales, económicas, físicas y ambientales?

Se considera que hay vulnerabilidad cuando ocurre un evento adverso y no se tiene la capacidad de respuesta ni la habilidad para adaptarse al nuevo escenario generado (Comisión Económica para América Latina y el Caribe [CEPAL], 2002). Esto se define por la interacción de una serie de factores internos y externos que confluyen hacia un individuo o grupo de personas, en un tiempo y espacio determinados (Busso, 2001). Aunque se presentan discrepancias en las definiciones de vulnerabilidad debido a las diferentes orientaciones epistemológicas y sus propias metodologías (Cutter, 1996), es claro que cuando la inversión social no llega a grupos específicos se generan situaciones de exclusión que repercuten en el rezago y el bienestar de las poblaciones, originando procesos diferenciales, complejos y heterogéneos (Vergara, 2011), e inestabilidades que se identifican en determinadas áreas (Armaș y Gavriș, 2013). Por lo tanto, la vulnerabilidad es una condición preexistente en las comunidades (Cutter y Emrich, 2006), con dominios espaciales y resultados variantes en localización y tiempo (Cutter, 1996), lo cual depende fundamentalmente del contexto, donde las diferencias sociales determinan posiciones de ventaja o desventaja ante el impacto potencial derivado de un fenómeno natural o antrópico. Además, la vulnerabilidad está influenciada por inequidades relacionadas con el lugar, como las características físicas de las comunidades y el ambiente, tanto natural como construido (Cutter, Boruff y Shirley, 2003). De esta manera, los lugares y personas pueden ser vulnerables debido a sus atributos biofísicos (Cutter, 1996) o condiciones físicas que influencian sus pérdidas potenciales y su capacidad de recuperación (Lawal y Arokoyu, 2015). Con base en lo anterior, la vulnerabilidad es un proceso multidimensional y multicausal, ligado a nociones de fragilidad e indefensión, desamparo institucional, debilidad interna, inseguridad, degradación, pobreza y exclusión social (Busso, 2005), susceptible a ser clasificada o desagregada en factores que la condicionan, para ser expresada en diferentes niveles de análisis (Busso, 2001). La Oficina de las Naciones Unidas para la Reducción del Riesgo de Desastres (UNISDR, 2004) clasificó la vulnerabilidad en cuatro dimensiones: social, económica, física y ambiental.

En el territorio, al ser un sistema socioambiental complejo (Bortoluzzi, Trevignani, D'Angelo, Mingiaca y Meriggiola, 2013), donde la distribución espacial de las desigualdades se encuentra condicionada por factores sociales, económicos, demográficos, naturales y culturales (Fuenzalida, 2015), las diferentes dimensiones interactúan para producir la vulnerabilidad general de dicho territorio, la cual es heterogénea espacialmente (Cutter, Boruff y Shirley, 2003). Aunque la vulnerabilidad no es un proceso teórico y metodológico único, ya que no es fácil medirla y observarla (Huang, Su, y Zhang, 2015), requiere combinar metodologías cuantitativas y cualitativas para su abordaje (Bus- 
so, 2005), enfocadas en la integración espacial sistemática de diversos componentes para su evaluación y comprensión (Burton y Cutter, 2008). Para ello, es indispensable el empleo de técnicas analíticas, con el fin de estudiar el orden espacial y las posibles aglomeraciones de las variables de estudio (Haki, 2003), así como proponer soluciones o alternativas de cuantificación útiles y accesibles a los tomadores de decisiones (Armaș y Gavriș, 2013). Una de estas alternativas se da a través de los índices de vulnerabilidad, herramientas que miden diferentes factores seleccionados, de acuerdo a su apoyo al concepto, en términos de validez, calidad y disponibilidad de los datos, así como a su simplicidad, reconocimiento y objetividad (Dwyer, Zoppou, Nielsen, Day y Roberts, 2004). Actualmente hay varias tendencias o enfoques en la construcción de índices de vulnerabilidad, que incluyen el análisis de escalas, la transformación y reducción de datos, la normalización, la ponderación y la agregación (Tate, 2012), donde el enfoque espacial "ofrece la oportunidad de visualizar susceptibilidades para todos y facilita la exploración de las posibles intervenciones" (Kienberger, Lang y Zeil, 2009, p. 776). Tal es el caso del índice de vulnerabilidad social (SoVI) (Cutter, Boruff y Shirley, 2003), el índice multicriterio de vulnerabilidad social (SEVI) (Armaș y Gavriş, 2013) o el índice modificado de vulnerabilidad socio-ambiental (M-SEVI) (Norman et al., 2012). Además, incorporar el análisis exploratorio de datos espaciales permite encontrar patrones y relaciones ocurrentes en el espacio (Haki, Akyürek y Düzgün, 2004), evaluando la vulnerabilidad y sus causas bajo el precepto de la autocorrelación espacial, es decir, similitudes en los valores y sus ubicaciones (Vergara, 2011).

En Colombia no son muchos los estudios que abordan la vulnerabilidad bajo el enfoque dimensional y espacial. Una primera aproximación consistió en determinar la vulnerabilidad sociodemográfica en el departamento de Cundinamarca de acuerdo a tres componentes: 1) condiciones de las viviendas, 2) situación de la población y 3) salud, y se encontraron altos índices de vulnerabilidad en los municipios ubicados en los límites del departamento debido a problemas relacionados con las viviendas, el empleo, la educación y la violencia (Castrillón, 2009). Otro estudio abarcó la definición de la vulnerabilidad en dos dimensiones: 1) sociodemográfica y económica, compuesta por quince variables; y 2) ambiental y geográfica, con cinco variables. A través del modelado cartográfico con herramientas de sistemas de información geográfica (SIG), se estableció una clasificación de vulnerabilidad socioambiental municipal representada a través de una cobertura tipo ráster, en la cual se destacó la alta vulnerabilidad al occidente del país (Chocó, Cauca y Nariño y sur del Tolima), y la baja vulnerabilidad en la Orinoquía (Arauca y Casanare) y gran parte de la región Caribe (Durán, 2012).

Bajo esta perspectiva teórica, se desarrolló un análisis espacial para la medición y categorización, por medio de índices, de la vulnerabilidad, tanto en sus diferentes dimensiones (social, económica, física y ambiental) como en general. El objetivo principal de este trabajo fue determinar la vulnerabilidad del territorio colombiano desde la conjugación de factores condicionantes, y así poder establecer zonas con mayores o menores fragilidades territoriales, bajo la hipótesis de que la vulnerabilidad multidimensional presenta distribuciones, comportamientos y patrones particulares basados en las condiciones sociales, económicas, físicas y ambientales que son intrínsecas de los territorios. Así, este trabajo busca convertirse en una herramienta de soporte necesaria para la comprensión y la evaluación del impacto de la vulnerabilidad ante posibles amenazas naturales y antrópicas y su comportamiento diferencial sobre el territorio, fundamental para una acertada toma de decisiones e intervenciones focalizadas. Posterior a esta introducción, el artículo establece la metodología fundamentada en el análisis espacial, que incluye la definición de factores y dimensiones, la exploración de datos espaciales, la creación de índices y la visualización. A continuación se presentan y analizan los resultados obtenidos y, por último, se exponen las conclusiones y la discusión. 


\section{Metodología}

Bajo la premisa de que el análisis espacial es una "colección de procesos con los que explotar los datos espaciales" (Olaya, 2014, p. 231), este trabajo se enmarca en la propuesta metodológica Problema, Plan, Datos, Análisis y Conclusiones (PPDAC), que es un método flexible y adaptable a las investigaciones socioeconómicas y ambientales (De Smith, Goodchild y Longley, 2015), e incluye los siguientes procesos:

\subsection{Selección de factores}

La definición de las dimensiones y sus factores incidentes se basa en el enfoque deductivo o a priori, de acuerdo con los fundamentos teóricos de la vul- nerabilidad (Tate, 2012). Con base en datos obtenidos de fuentes oficiales, se realizó la selección de acuerdo a la revisión de trabajos conceptuales, analíticos y aplicados al tema de la vulnerabilidad, lo que permite establecer su validez y objetividad al considerar el criterio experto. Cada factor está definido por una variable medida a través de un indicador, el cual está calculado para cada uno de los 1.122 municipios del país (que incluyen las veinte áreas no municipalizadas) como unidad de análisis, con el fin de garantizar la misma escala geográfica, así como una mayor cobertura y desagregación de la información. En las Tablas 1 a 4 se listan los factores por cada dimensión, su indicador, el año y el tipo de relación con la vulnerabilidad, ya sea directa o inversa, de acuerdo a su medición.

\begin{tabular}{|c|c|c|c|c|}
\hline Variable & Indicador & Fuente & Año & Relación \\
\hline Adultos mayores & Porcentaje de población mayor de 60 años & DANE $^{2}$ & 2013 & Directa \\
\hline Niñez & Porcentaje de población menor de 12 años & DANE & 2013 & Directa \\
\hline Mortalidad & Tasa de mortalidad infantil & DANE & 2013 & Directa \\
\hline Educación & Porcentaje de cobertura en educación media neta & $\mathrm{MEN}^{3}$ & 2013 & Inversa \\
\hline Participación & Porcentaje de participación electoral & $\mathrm{RN}^{4}$ & 2011 & Inversa \\
\hline Ruralidad & Porcentaje de población en resto rural & DANE & 2014 & Directa \\
\hline Etnicidad & Porcentaje de área en territorios étnicos & $I_{G A C^{5}}$ & 2014 & Directa \\
\hline Discapacidad & Porcentaje de personas en condición de discapacidad & DANE & 2010 & Directa \\
\hline Desplazamiento forzado & Índice de intensidad de desplazamiento & $\mathrm{UV}^{6}$ & 2013 & Directa \\
\hline Pobreza & Porcentaje de población con necesidades básicas insatisfechas & DANE & 2012 & Directa \\
\hline
\end{tabular}

TABLA 2. DESCRIPCIÓN DE LOS FACTORES EN LA VULNERABILIDAD ECONÓMICA

\begin{tabular}{lllll} 
Variable & Indicador & Fuente & Año & Relación \\
\hline Inversión & Inversión per cápita en el sector ambiental & DNP $^{7}$ & 2012 & Inversa \\
\hline Ingresos & Ingreso tributario per cápita & DNP & 2012 & Inversa \\
\hline Desempeño & Indicador de desempeño integral & DNP & 2013 & Inversa \\
\hline Valoración & Promedio del valor predial & IGAC & 2014 & Inversa
\end{tabular}

Fuente: Elaboración propia.

Departamento Administrativo Nacional de Estadística

Ministerio de Educación Nacional

Registraduría Nacional del Estado Civil

Instituto Geográfico Agustín Codazzi

Unidad para las Víctimas

Departamento Nacional de Planeación 
TABLA 3. DESCRIPCIÓN DE LOS FACTORES EN LA VULNERABILIDAD FÍSICA

\begin{tabular}{lllll}
\hline Variable & Indicador & Fuente & Año & Relación \\
\hline Drenajes & Densidad de drenajes & IGAC & 2014 & Inversa \\
\hline Densidad de población & Densidad de población & DANE & 2014 & Directa \\
\hline Sismología & Número de sismos registrados & SGC & $2013-2014$ & Directa \\
\hline Fallas geológicas & Longitud de fallas geológicas & SGC & 2015 & Directa \\
\hline Vías & Densidad de vías & IGAC & 2014 & Inversa \\
\hline Fuente: Elaboración propia. & & & &
\end{tabular}

TABLA 4. DESCRIPCIÓN DE LOS FACTORES EN LA VULNERABILIDAD AMBIENTAL

\begin{tabular}{lllcc}
\hline Variable & Indicador & Fuente & Año & Relación \\
\hline Temperatura & Temperatura media anual & IDEAM $^{9}$ & 1981-2010 & Directa \\
\hline Precipitaciones & Precipitación anual & IDEAM & $1981-2010$ & Directa \\
\hline Calidad del agua & Índice de calidad del agua & IDEAM & 2011 & Inversa \\
\hline Calidad del suelo & $\begin{array}{l}\text { Porcentaje de área en erosión o desertificación moderada } \\
\text { a muy severa }\end{array}$ & IDEAM & 2012 & Directa \\
\hline Áreas protegidas & Porcentaje de áreas protegidas & IGAC - HB ${ }^{10}$ & 2013 & Directa \\
\hline
\end{tabular}

Fuente: Elaboración propia.

\subsection{Análisis exploratorio de datos espaciales}

Este enfoque analítico permite describir y visualizar las distribuciones de los datos, identificar atípicos y descubrir asociaciones o agrupamientos que sugieren algún tipo de estructura o heterogeneidad espacial (Anselin, 1998). Actualmente, el análisis exploratorio de datos espaciales (AEDE) es una herramienta relevante en el marco de las políticas públicas y sociales, ya que permite dilucidar las diferentes desigualdades presentes en los territorios (Fuenzalida, 2015). Con base en lo anterior, se realiza inicialmente el análisis individual de los factores a través de herramientas estadísticas. A continuación, bajo el supuesto de que los datos se distribuyen en el espacio de manera aleatoria, se busca establecer si los valores de cada variable en un municipio tienen relación directa con otros municipios cercanos, lo que permite identificar patrones espaciales. Para ello se calcula el estadístico espacial $I$ de Moran, que junto con el Z-score y el p-valor determinarán el nivel de confianza que permita confirmar o rechazar la hipótesis inicial. La representación espacial de los patrones encontrados se realiza a través del indicador local de asociación espacial Anselin Local Moran; esta es una parte crucial del análisis en la presentación y comunicación de resultados intermedios en el estudio (Bivand, 2010), ya que "cualquier metodología para el análisis de datos espaciales necesita reconocer que estos tienen la propiedad fundamental de la dependencia espacial o autocorrelación espacial" (Haining, Kerry y Oliver, 2010, p. 8).

8 Servicio Geológico Colombiano

9 Instituto de Hidrología, Meteorología y Estudios Ambientales

10 Instituto de Investigación de Recursos Biológicos Alexander Von Humboldt

Vol. 22 N. ${ }^{\circ} 1$ 


\subsection{Construcción de índices de vulnerabilidad y visualización}

Un índice compuesto es una agregación de indicadores individuales agrupados por dimensiones, que tiene como objetivo proporcionar una medida sintética de un problema complejo, con base en la transformación de información que permita comparar valores en una escala fija (United States Agency for International Development [USAID], 2014). Dado que los indicadores tienen diferentes escalas de medida, se realiza la normalización de los valores por medio del Z-score, el cual es un método de estandarización apropiado para series de datos con valores extremos (Nardo, Saisana, Saltelli y Tarantola, 2005). De acuerdo al enfoque analítico basado en la autocorrelación espacial, se emplea como valor normalizado el Z-score obtenido con el estadístico espacial local Getis-Ord Gi*, el cual distingue las concentraciones de valores bajos (Z-score negativo) y valores altos (Z-score positivo) (Wang y Wong, 2011). Los factores seleccionados adquieren el mismo peso en el cálculo de los índices ya que, al ser obtenidos de fuentes secundarias, no se conocen plenamente los procesos subyacentes que permiten derivar ponderaciones diferenciadas (Cutter, Boruff y Shirley, 2003), lo que podría conducir a una asignación de pesos no justificada (USAID, 2014). La agregación de los factores normalizados se hace a través de la sumatoria de los mismos, debido a su simplicidad, facilidad de comprensión y aplicación general (Nardo et al., 2005). De esta manera, para cada uno de los municipios se obtienen los valores de cada índice por dimensión, que al ser adicionados determinan la vulnerabilidad total.

La visualización de la vulnerabilidad a través de la representación cartográfica permite comunicar puntos clave en su distribución geográfica, así como percibir, comparar y evaluar patrones espaciales presentes en diferentes ubicaciones (Martin, 2005). Por lo tanto, los diferentes índices de vul- nerabilidad se visualizan a través de mapas temáticos para representar su comportamiento espacial, empleando gamas de color bivariadas, ya que los índices presentan un punto de inflexión entre valores negativos (menor vulnerabilidad) y positivos (mayor vulnerabilidad) (Aránguez, Arribas, Aránguez y Ordóñez, 2012).

\section{Resultados y análisis}

\subsection{Análisis exploratorio de datos espaciales}

Algunas variables presentan distribuciones con poca asimetría. En la dimensión social, la media del porcentaje de menores es de $26,3 \%$ y la de adultos mayores $11,1 \%$; en cobertura de educación media es del $35 \%$, mientras que en porcentaje de población con necesidades básicas insatisfechas (NBI) es del $45 \%$. En la dimensión ambiental, las precipitaciones se agrupan en valores cercanos a los $2.000 \mathrm{~mm}$, la calidad del agua promedia en el rango de clasificación regular, y la temperatura media se concentra sobre los $20^{\circ} \mathrm{C}$.

Las fuertes asimetrías positivas indican una baja participación de personas en condiciones particulares de discapacidad o desplazamiento, así como prevalecen las tasas de mortalidad infantil inferiores al 20 \%. En la dimensión económica, el sesgo presente en las variables ingreso, inversión y valor promedio ratifica que cerca del $90 \%$ de los municipios del país son de sexta categoría, es decir, municipios con menores ingresos de libre destinación. Así mismo, no son muchos los municipios que presentan condiciones considerables de inestabilidad geológica o sismicidad. En contraste, las asimetrías negativas indicarían una tendencia superior al $60 \%$ de participación electoral en los municipios, y un desempeño institucional que se agrupa sobre calificaciones satisfactorias (alrededor del valor 70). 
Se presentan datos atípicos extremos en la tasa de mortalidad infantil (TMI), la cual se estima a través de modelos de regresión de las estadísticas vitales (nacimientos y defunciones); la obtención de estos registros puede tener limitaciones en las zonas donde prevalecen valores muy altos ${ }^{11}$. Sin embargo, al no tener certeza de los lugares donde ocurren estos hechos y su verdadero impacto, no se removerán del análisis. En el caso de la población adulta mayor, se presenta un valor atípico superior respaldado por una característica particular del municipio de Guayatá (Boyacá), considerado el "pueblo de los viejos"12. Los atípicos extremos inferiores en la participación electoral (valores cero) son consecuencia de una posible omisión en la captura de información, y serán removidos del análisis. El índice de desempeño integral con valores inferiores a 40 incluye municipios sin in- formación o no evaluables por inconsistencias ${ }^{13}$, lo cual indica la posibilidad de datos no aptos para el análisis, que están por el orden del valor 10, de acuerdo al diagrama de caja. La precipitación anual en Colombia cuenta con una de las regiones más lluviosas del mundo, la cuenca del Alto Patía, lo cual justifica la presencia de valores extremos por este fenómeno.

Todos los factores analizados presentan autocorrelación espacial positiva o patrón de concentración, es decir, agrupaciones espaciales de valores altos o bajos, confirmada por $I$ de Moran mayor a cero y cercano a 1, rechazando el supuesto de aleatoriedad con un nivel de confianza superior al $99 \%$, gracias a los valores muy altos del Z-score (superiores a 2,58) y muy pequeños del p-valor (cercanos o iguales a cero) (Tabla 5).

\section{TABLA 5. ÍNDICE DE MORAN, Z-SCORE Y P-VALOR EN ORDEN DESCENDENTE}

\begin{tabular}{lllll} 
Variable & Dimensión & I de Moran & Z-score & P-valor \\
\hline Temperatura & Ambiental & 0,893721 & 51,288406 & 0,000000 \\
\hline Índice de calidad del agua & Ambiental & 0,855291 & 49,104412 & 0,000000 \\
\hline Precipitación & Ambiental & 0,753111 & 43,368825 & 0,000000 \\
\hline Territorio étnico & Social & 0,681667 & 39,313018 & 0,000000 \\
\hline Densidad de vías & Física & 0,612250 & 35,186473 & 0,000000 \\
\hline Necesidades básicas insatisfechas & Social & 0,610748 & 35,082127 & 0,000000 \\
\hline Población menor & Social & 0,573715 & 32,961086 & 0,000000 \\
\hline Erosión o desertificación & Ambiental & 0,558180 & 32,049459 & 0,000000 \\
\hline Población adulto mayor & Social & 0,550460 & 31,638564 & 0,000000 \\
\hline Tasa de mortalidad infantil & Social & 0,547340 & 31,581090 & 0,000000 \\
\hline Participación electoral & Social & 0,518669 & 29,310043 & 0,000000 \\
\hline Áreas protegidas & Ambiental & 0,418663 & 24,108016 & 0,000000 \\
\hline Fallas geológicas & Física & 0,401950 & 23,291286 & 0,000000 \\
\hline Índice de desempeño integral & Económica & 0,397130 & 22,633596 & 0,000000 \\
\hline Cobertura en educación media & Social & 0,377830 & 21,550829 & 0,000000 \\
\hline Valoración predial & Económica & 0,372624 & 22,858020 & 0,000000 \\
\hline Población desplazada & Social & 0,341393 & 21,064504 & 0,000000 \\
\hline
\end{tabular}

11 Posible omisión, en algunos departamentos, de hechos vitales que ocurren fuera del sistema de salud y de otros, a causa de la gran dispersión del territorio y el no reporte por condiciones culturales (http://www.dane.gov.co/files/investigaciones/poblacion/ vitales/nota_metodologica.pdf).

12 En el municipio de Guayatá, en los últimos años se han cerrado dos escuelas veredales por falta de estudiantes, y se imponen los programas para salvaguardar a la tercera edad (http://www.eltiempo.com/archivo/documento/MAM-4930755).

13 Evaluación del desempeño integral de los municipios y distritos, vigencia 2013 (Departamento Nacional de Planeación - Dirección de Desarrollo Territorial Sostenible).

Vol. 22 N. ${ }^{\circ} 1$ 


\begin{tabular}{lllll} 
Variable & Dimensión & I de Moran & Z-score & P-valor \\
\hline Ingreso tributario per cápita & Económica & 0,337274 & 19,806120 & 0,000000 \\
\hline Densidad de drenajes & Física & 0,321871 & 19,068242 & 0,000000 \\
\hline Población en discapacidad & Social & 0,307516 & 17,730335 & 0,000000 \\
\hline Población rural & Social & 0,306372 & 17,619040 & 0,000000 \\
\hline Densidad de población & Física & 0,285202 & 18,583881 & 0,000000 \\
\hline Inversión ambiental per cápita & Económica & 0,214408 & 12,658475 & 0,000000 \\
\hline Número de sismos & Física & 0,077239 & 18,196911 & 0,000000
\end{tabular}

Para distinguir la presencia de agrupaciones o clústeres a nivel local, se representan en color rojo aquellos patrones que influyen directamente en los distintos tipos de vulnerabilidad, y en color azul, la relación inversa. Así mismo, se destaca la presencia de atípicos espaciales: valor alto rodeado de bajos y valor bajo rodeado de altos.

En la dimensión social, coinciden los patrones que concentran alta población infantil y desplazada, bajas coberturas de educación media, altas NBI (Figura 1) y TMI y baja participación electoral en las áreas donde predominan territorios étnicos

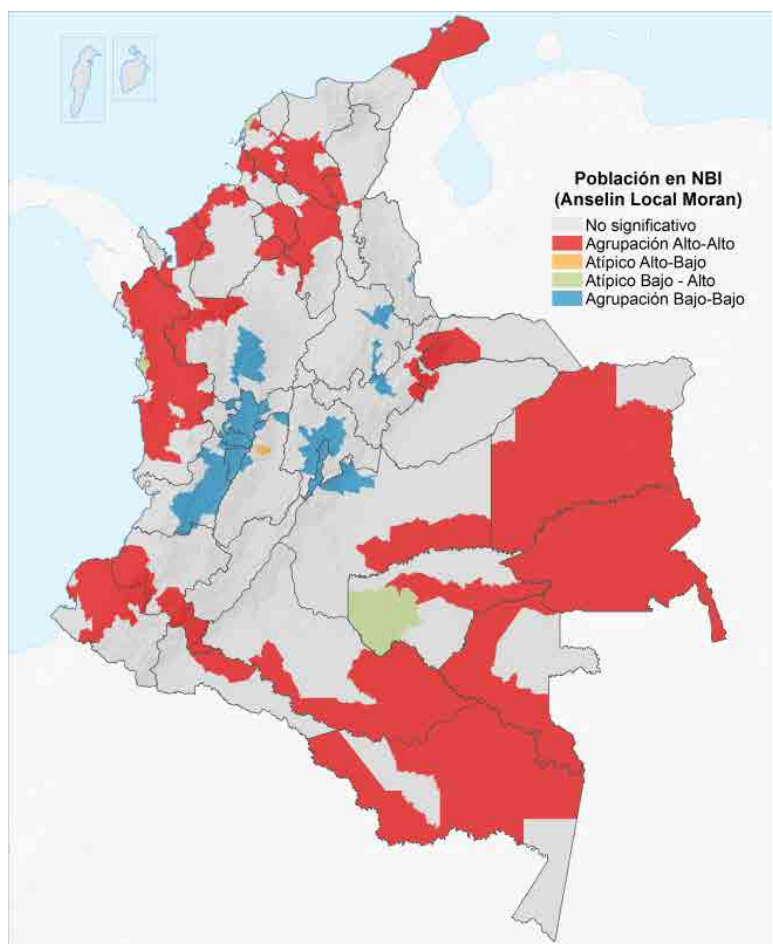

(alta Guajira, andén Pacífico, Orinoquía y Amazonía). Por consiguiente, en estas zonas periféricas del país se conjugan factores condicionantes de la exclusión social. En la dimensión económica, a pesar de encontrarse patrones de concentración en todas las variables, estos no se manifiestan de forma significativa a nivel local, salvo en el índice de desempeño integral (IDI), que agrupa valores bajos esencialmente en la región Caribe y Vichada (Figura 2), lo que representa una baja capacidad institucional que influye en una menor acción y atención ante distintos tipos de amenazas.
Figura 1. Patrones locales de NBI.

Fuente: Elaboración propia. 


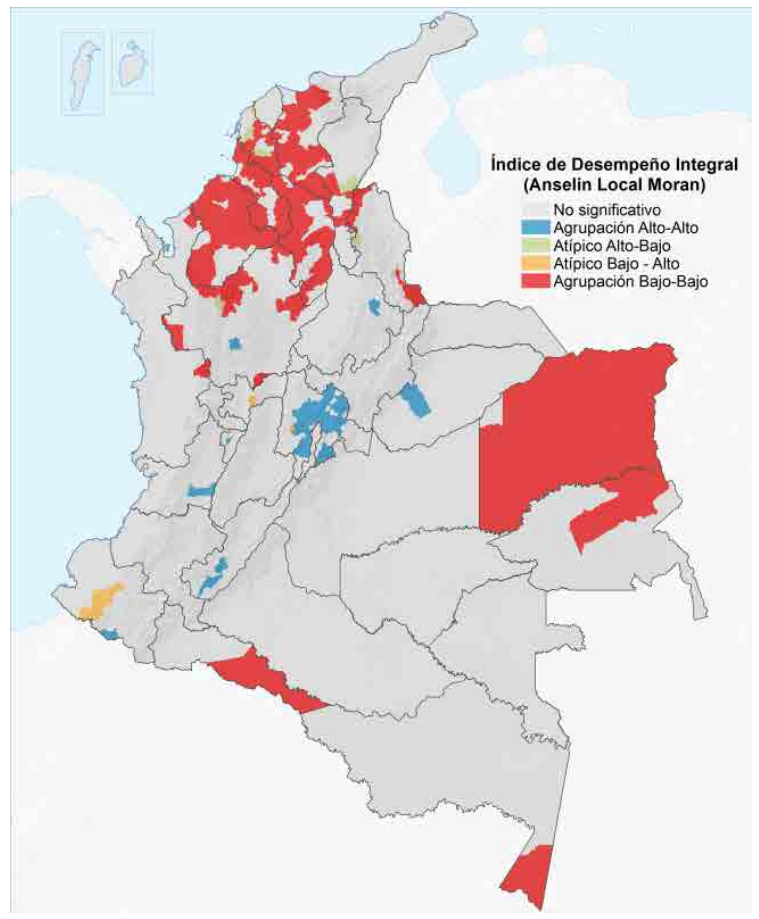

Figura 2. Patrones locales de IDI.

Fuente: Elaboración propia.

En la dimensión física se destaca la baja densidad de vías en las zonas periféricas del país, altas longitudes de fallas geológicas en el occidente del territorio nacional (Figura 3), y alto número de sismos concentrados específicamente en la zona de la Mesa de los Santos (Santander). Esto demuestra que una limitada infraestructura vial, que impide el acceso pleno a bienes y servicios, amplía las brechas sociales ya marcadas anteriormente entre el centro y la periferia, y esto se suma a las dificultades propias de la inestabilidad geológica. Para las variables de la dimensión ambiental, la baja erosión o desertificación (Figura 4) se concentra en lugares donde la calidad del agua es alta y las precipitaciones son significativas, principalmente en el andén Pacífico y la Amazonía, es decir, donde predomina el bosque tropical húmedo y el impacto antrópico ha sido a menor escala.
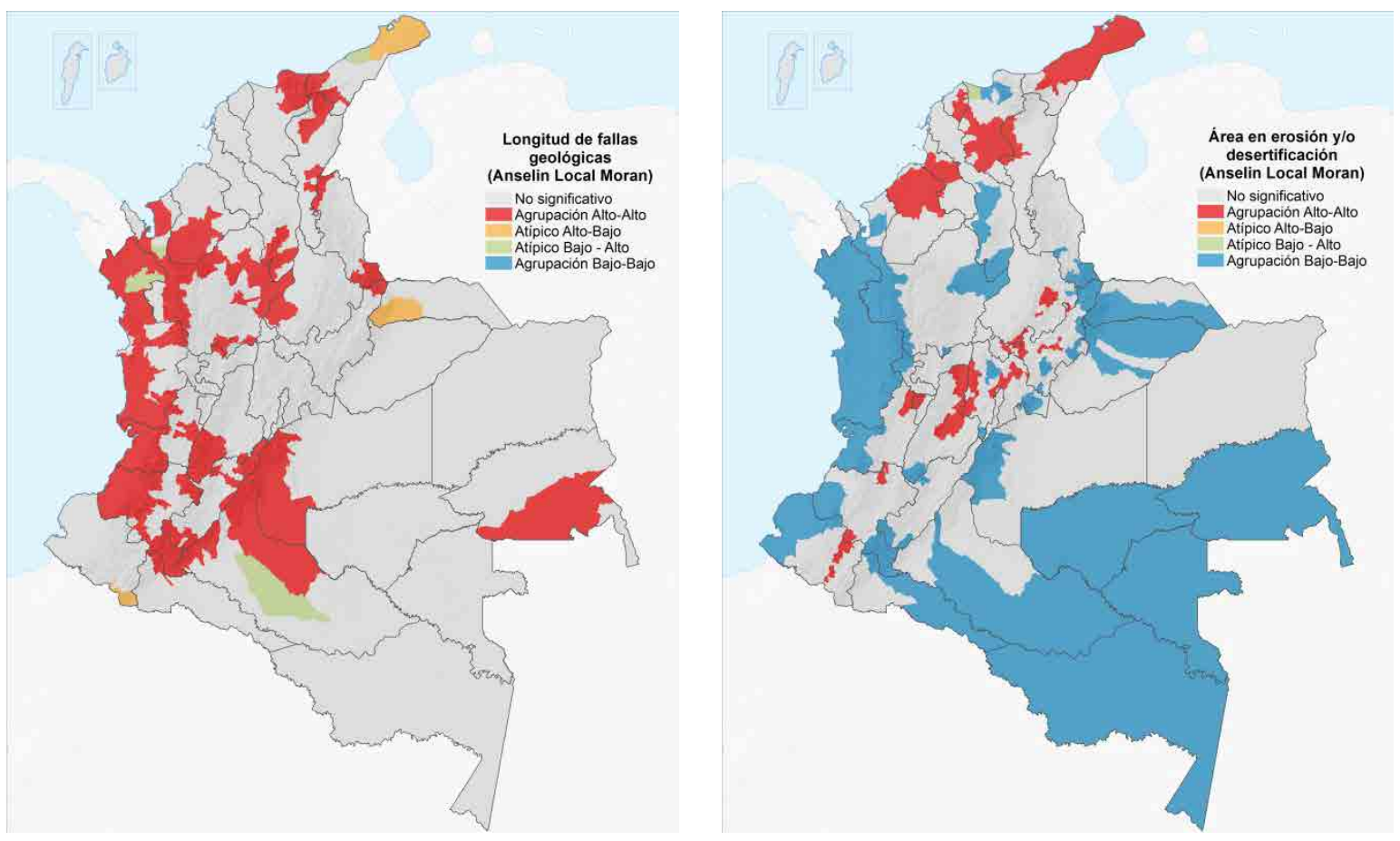

Figura 3. Patrones locales de fallas geológicas.

Fuente: Elaboración propia.

Figura 4. Patrones locales de erosión.

Fuente: Elaboración propia. 


\section{2 Índices de vulnerabilidad y visualización}

Para cada dimensión de vulnerabilidad se genera un índice compuesto a través de la adición de cada uno de los factores equiponderados, donde la suma de pesos equivale al $100 \%$ del valor del índice (Tabla 6).

\begin{tabular}{lcc} 
& TABLA 6. PONDERACIÓN DE FACTORES POR CADA DIMENSIÓN \\
\multicolumn{1}{c}{ Dimensión } & Número de factores & Ponderación para cada factor \\
\hline Social & 10 & $10 \%$ \\
\hline Económica & 4 & $25 \%$ \\
\hline Física & 5 & $20 \%$ \\
\hline Ambiental & 5 & $20 \%$ \\
\hline
\end{tabular}

Fuente: Elaboración propia.

Índice de vulnerabilidad social (IVS)

IVS $=0,1$ (Adultos Mayores) $+0,1$ (Niñez) $+0,1(\mathrm{TMI})+0,1$ (Educación) $+0,1$ (Participación )+ 0,1 (Ruralidad) $+0,1$ (Etnicidad) $+0,1$ (Discapacidad) $+0,1$ (Desplazamiento) $+0,1$ (NBI)

Índice de vulnerabilidad económica (IVE)

IVE=0,25(Inversión)+0,25(Ingresos)+0,25(Desempeño)+0,25(Valoración)

Índice de vulnerabilidad física (IVF)

$\mathrm{IVF}=0,2$ (Drenajes) $+0,2$ (Densidad población) $+0,2$ (Sismología) $+0,2$ (Fallas geológicas) $+0,2($ Vías $)$

Índice de vulnerabilidad ambiental (IVA)

IVA $=0,2$ (Temperatura $)+0,2$ (Precipitación $)+0,2($ Calidad agua $)+0,2($ Calidad suelo $)+$ 0,2 (Áreas protegidas)

La vulnerabilidad total se determina mediante la adición equiponderada de cada uno de los ín-

Índice de vulnerabilidad total (IVT)

$\mathrm{IVT}=0,25(\mathrm{IVS})+0,25(\mathrm{IVE})+0,25(\mathrm{IVF})+0,25(\mathrm{IVA})$

La interpretación del valor del índice en cada una de las dimensiones se hace de manera similar al valor del Z-score: valores altos positivos (superiores a 1,65) indican una probabilidad significativa de alta vulnerabilidad, y bajos valores dices por dimensión calculados, de la siguiente manera:

negativos (inferiores a 1,65) una probabilidad significativa de baja vulnerabilidad. Las Tablas 7 a 11 destacan los cinco municipios con mayor y menor índice de vulnerabilidad por cada dimensión y total. 
TABLA 7. MUNICIPIOS CON MAYOR (IZQUIERDA) Y MENOR (DERECHA) ÍNDICE DE VULNERABILIDAD SOCIAL

\begin{tabular}{llllll} 
Municipio & Departamento & IVS & Municipio & Departamento & IVS \\
\hline Istmina & Chocó & 3,975 & Madrid & Cundinamarca & $-2,299$ \\
\hline Alto Baudó & Chocó & 3,330 & Funza & Cundinamarca & $-2,188$ \\
\hline Solano & Caquetá & 3,215 & Tenjo & Cundinamarca & $-2,164$ \\
\hline Bajo Baudó & Chocó & 3,102 & Chía & Cundinamarca & $-2,160$ \\
\hline El Charco & Nariño & 3,059 & Bogotá, D. C. & Cundinamarca & $-2,050$ \\
\hline
\end{tabular}

Fuente: Elaboración propia.

La alta incidencia de vulnerabilidad social se manifiesta principalmente en municipios de los departamentos de Chocó, Caquetá y Nariño, ratificando así las condiciones marginales y exclu- yentes presentes en las zonas periféricas del país. De otro lado, la baja vulnerabilidad sobresale al interior del país, en municipios que conforman conurbaciones alrededor del Distrito Capital.

TABLA 8. MUNICIPIOS CON MAYOR (IZQUIERDA) Y MENOR (DERECHA) ÍNDICE DE VULNERABILIDAD ECONÓMICA

\begin{tabular}{llllll} 
Municipio & Departamento & IVE & Municipio & Departamento & IVE \\
\hline Achí & Bolívar & 2,177 & Itagüí & Antioquia & $-8,118$ \\
\hline Montería & Córdoba & 2,153 & Envigado & Antioquia & $-7,960$ \\
\hline San Fernando & Bolívar & 2,147 & Sabaneta & Antioquia & $-6,545$ \\
\hline Pinillos & Bolívar & 2,122 & Cota & Cundinamarca & $-6,065$ \\
\hline Lorica & Córdoba & 2,101 & Chía & Cundinamarca & $-5,678$
\end{tabular}

Fuente: Elaboración propia.

La alta vulnerabilidad económica se manifiesta especialmente en municipios de los departamentos de Bolívar y Córdoba, mientras que la baja vulnerabilidad es significativa en municipios de los departamentos de Antioquia y Cundinamarca. Estos resultados muestran un patrón de menor ca- pacidad institucional en la captación e inversión de recursos en la región Caribe, en contraste con mejores índices de eficacia-eficiencia y una alta valoración predial de los municipios colindantes o áreas metropolitanas en las grandes ciudades del país.

\section{TABLA 9. MUNICIPIOS CON MAYOR (IZQUIERDA) Y MENOR (DERECHA) ÍNDICE DE VULNERABILIDAD FÍSICA}

\begin{tabular}{llllll} 
Municipio & Departamento & IVF & Municipio & Departamento & IVF \\
\hline Itagüí & Antioquia & 3,034 & Santo Tomás & Atlántico & $-2,750$ \\
\hline Zapatoca & Santander & 2,805 & Polonuevo & Atlántico & $-2,556$ \\
\hline Uribe & Meta & 2,644 & Suan & Atlántico & $-2,491$ \\
\hline Los Santos & Santander & 2,630 & Campo Cruz & Atlántico & $-2,461$ \\
\hline Jordán & Santander & 2,616 & Sabanagrande & Atlántico & $-2,423$ \\
\hline
\end{tabular}

Fuente: Elaboración propia.

La alta sismicidad en determinados municipios de Santander repercute en una mayor vulnerabilidad física. Lo mismo ocurre donde la densidad de población es muy alta, como en Itagüí. Ahora bien, la disponibilidad de grandes fuentes hídricas superficiales en los municipios del Atlántico podría mitigar el impacto ante una eventual escasez del recurso agua . 
TABLA 10. MUNICIPIOS CON MAYOR (IZQUIERDA) Y MENOR (DERECHA) ÍNDICE DE VULNERABILIDAD AMBIENTAL

\begin{tabular}{llllll} 
Municipio & Departamento & IVA & Municipio & Departamento & IVA \\
\hline Istmina & Chocó & 2,750 & San Agustín & Huila & $-2,885$ \\
\hline Cértegui & Chocó & 2,574 & Socotá & Boyacá & $-2,703$ \\
\hline C. San Pablo & Chocó & 2,494 & Santa Rosa & Cauca & $-2,695$ \\
\hline Alto Baudó & Chocó & 2,405 & Fómeque & Cundinamarca & $-2,615$ \\
\hline Río Quito & Chocó & 2,248 & Pitalito & Huila & $-2,587$ \\
\hline
\end{tabular}

Fuente: Elaboración propia.

Las condiciones de extrema pluviosidad repercuten significativamente en la alta vulnerabilidad ambiental, principalmente en municipios del departamento de Chocó. En contraste, los municipios con temperaturas medias, calidad del agua aceptable, suelos sin degradación y áreas destinadas a la protección ambiental reflejan una menor vulnerabilidad.

\begin{tabular}{|c|c|c|c|c|c|}
\hline Municipio & Departamento & IVT & Municipio & Departamento & IVT \\
\hline Istmina & Chocó & 2,317 & Chía & Cundinamarca & $-2,413$ \\
\hline Alto Baudó & Chocó & 2,004 & Cota & Cundinamarca & $-2,239$ \\
\hline Quibdó & Chocó & 1,860 & Funza & Cundinamarca & $-2,093$ \\
\hline Bajo Baudó & Chocó & 1,858 & Zipaquirá & Cundinamarca & $-2,028$ \\
\hline C. San Pablo & Chocó & 1,708 & Sopó & Cundinamarca & $-1,986$ \\
\hline
\end{tabular}

Los valores más altos del índice de vulnerabilidad total (IVT) en los municipios de Chocó reflejan marcadas condiciones de exclusión social, baja captación e inversión de recursos, inestabilidades geológicas, poca o nula infraestructura y pluviosidad extrema. En contraste, los municipios de Cundinamarca cercanos a Bogotá, D. C., influenciados por su dinamismo socioeconómico, que perciben altos ingresos y con alta infraestructura vial gracias a su ubicación sobre el paisaje estable de la sabana o altiplano, generan condiciones que garantizan una reducción de la vulnerabilidad y, por lo tanto, escenarios más favorables ante una posible amenaza.

La representación de cada índice a través de mapas temáticos presenta de manera más clara los patrones espaciales discutidos, tanto de alta como de baja vulnerabilidad.

La vulnerabilidad social (Figura 5) refleja patrones claros de alta incidencia en Chocó, el andén Pacífico de Cauca y Nariño, la Amazonía y la alta
Guajira, con susceptibilidad de propagación hacia la Orinoquía en el sur, el bajo Cauca antioqueño, el sur de Bolívar y la región del Catatumbo en el norte, caso contrario a Bogotá, D. C. y Medellín, y en menor medida, Cali, Barranquilla y Bucaramanga, que junto a determinados municipios colindantes se caracterizan por ser polos de desarrollo con mejores condiciones sociales. Se manifiesta, por lo tanto, una clara delimitación que resalta las brechas establecidas entre el centro y la periferia.

En la vulnerabilidad económica (Figura 6) se manifiesta un patrón de alta incidencia en Córdoba, Sucre, Bolívar y Magdalena, influenciado principalmente por un bajo desempeño institucional. De otro lado, Bogotá, D.C., Medellín y Bucaramanga, con sus zonas de influencia, junto al sur de Casanare, el nororiente del Meta y el archipiélago de San Andrés y Providencia presentan patrones de baja vulnerabilidad, ya que disponen de importantes fuentes de ingresos propios, así como transferencias y regalías, y esto se suma a una alta valoración predial y capacidad tributaria. 
Para la vulnerabilidad física (Figura 7), los patrones de alta incidencia se manifiestan en el andén Pacífico, el noroccidente Antioqueño, la Mesa de los Santos (Santander) y la zona limítrofe de Meta y Caquetá, con presencia de alta inestabilidad geológica y sismicidad. La baja vulnerabilidad se manifiesta de manera tenue en el Altiplano Cundiboyacense, Atlántico y los límites entre Cundinamarca y Tolima, gracias a una buena infraestructura vial.

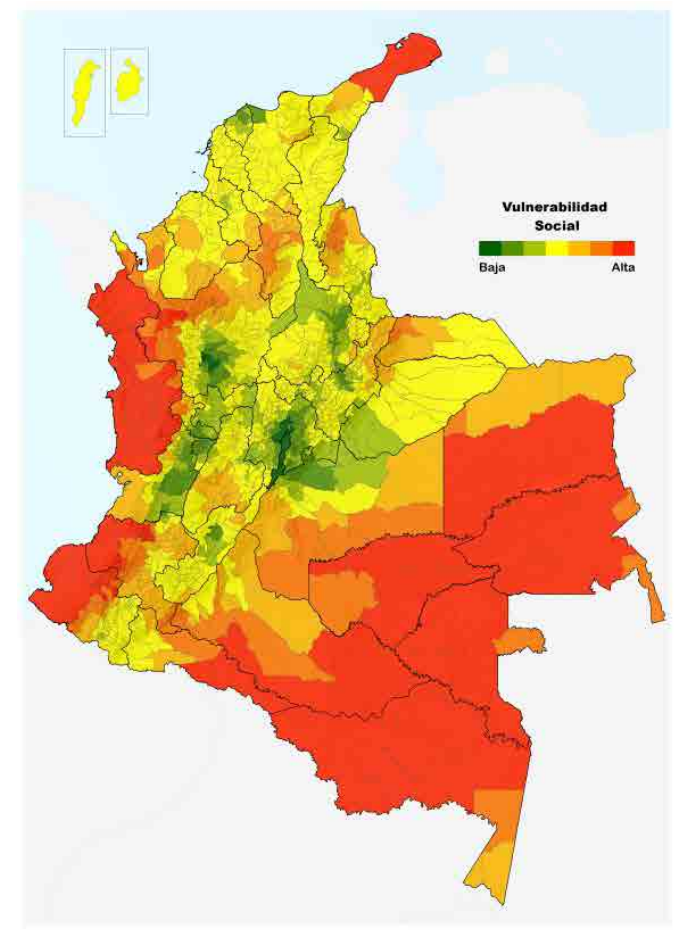

Figura 5. Vulnerabilidad social.

Fuente: Elaboración propia.
La vulnerabilidad ambiental (Figura 8) refleja una alta incidencia en Chocó, con significativa pluviosidad; en los límites de Cesar y Magdalena, con temperaturas altas por encima del promedio, así como en el trayecto medio del río Magdalena, donde predomina la mala calidad del agua. En la región Andina, principalmente en el macizo colombiano y la cordillera Oriental, se manifiesta una vulnerabilidad baja debido a las temperaturas templadas, con niveles moderados de precipitación, y la presencia de áreas protegidas tipo páramo.

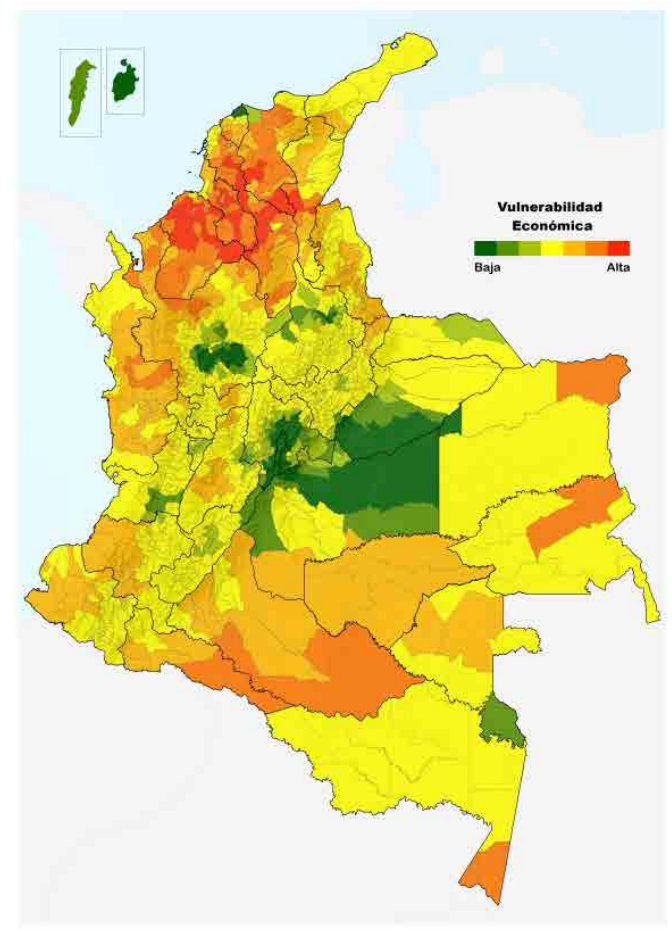

Figura 6. Vulnerabilidad económica.

Fuente: Elaboración propia. 


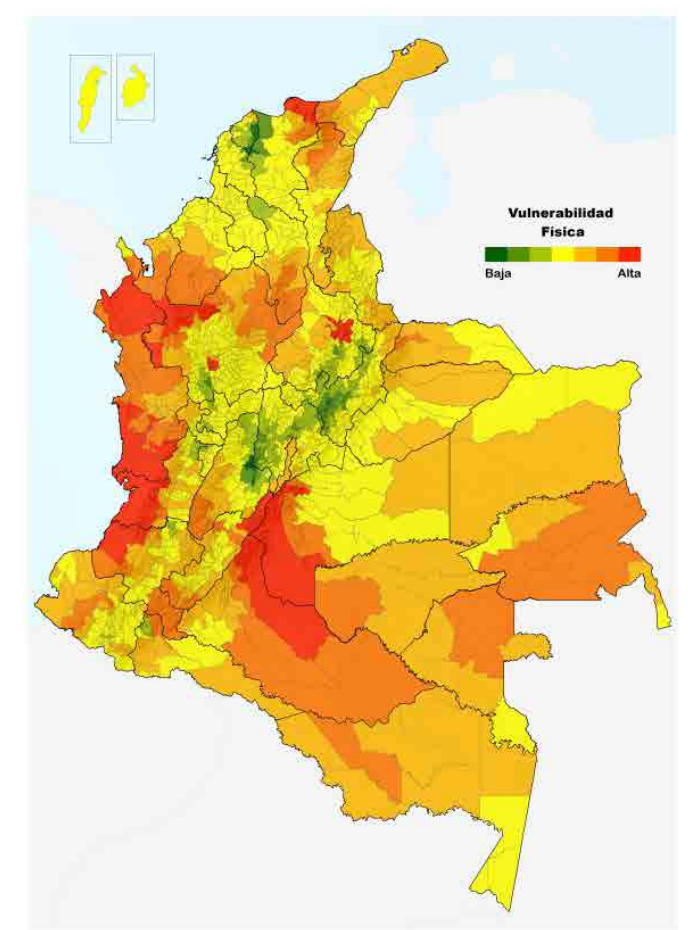

Figura 7. Vulnerabilidad física.

Fuente: Elaboración propia.

La vulnerabilidad total del territorio (Figura 9), que conjuga todas las dimensiones, revela una alta incidencia predominante en Chocó, con probabilidad de expansión hacia el resto del andén Pacífico, el bajo Cauca, el Urabá antioqueño y el sur de Bolívar. De igual manera, se exhibe una tendencia similar en la Amazonía y parte de la Orinoquía. En las zonas mencionadas, los municipios se caracterizan por tener una alta población rural y étnica, una baja cobertura en educación y poca participación electoral, así como influencia directa del conflicto armado lo que deriva en altos niveles de mortalidad infantil, pobreza y desplazamientos forzados, afianzados por los escasos recursos, la reducida capacidad institucional, los ingresos escasos y la poca inversión. Esto, sumado a los entornos inestables físicamente, la baja o nula infraestructura vial y las condiciones ambientales de alta temperatura y precipitación elevada, conforman un conjunto espacial claramente demarcado de fragilidades territoriales que aumentan la pro-

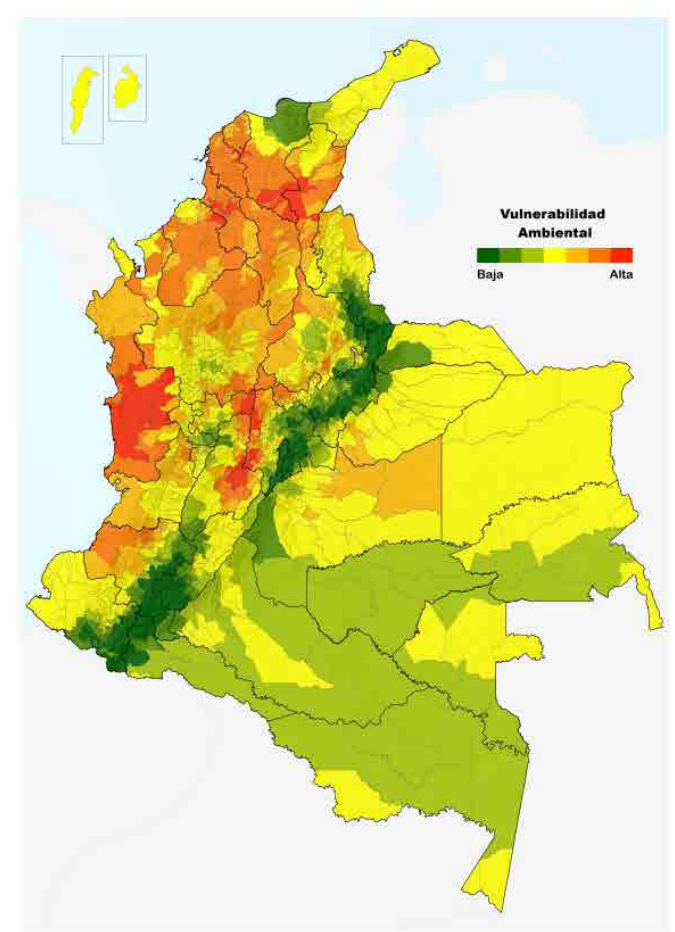

Figura 8. Vulnerabilidad ambiental,

Fuente: Elaboración propia.

babilidad de pérdidas y menor capacidad de prevención-mitigación ante cualquier amenaza, sea esta de origen natural o antrópico.

De otro lado, las zonas de influencia de los denominados "polos de desarrollo", principalmente Bogotá, D. C. y el Altiplano Cundiboyacense, Medellín, con su área metropolitana, y aquellas regiones donde se manifiesta una importante actividad económica relacionada con la producción petrolera (Meta y Casanare), representan agregaciones de baja incidencia a la vulnerabilidad. Esto sugiere que una mayor actividad productiva, con posibilidades de acceso a servicios básicos, alta participación electoral, mayor capacidad de gestión de recursos y demanda significativa en el mercado inmobiliario, que junto a una mejor infraestructura construida, geología estable y sin condiciones climáticas extremas, establecen condiciones más adecuadas para el soporte y la adaptación a un nuevo escenario surgido ante la materialización de una amenaza. 


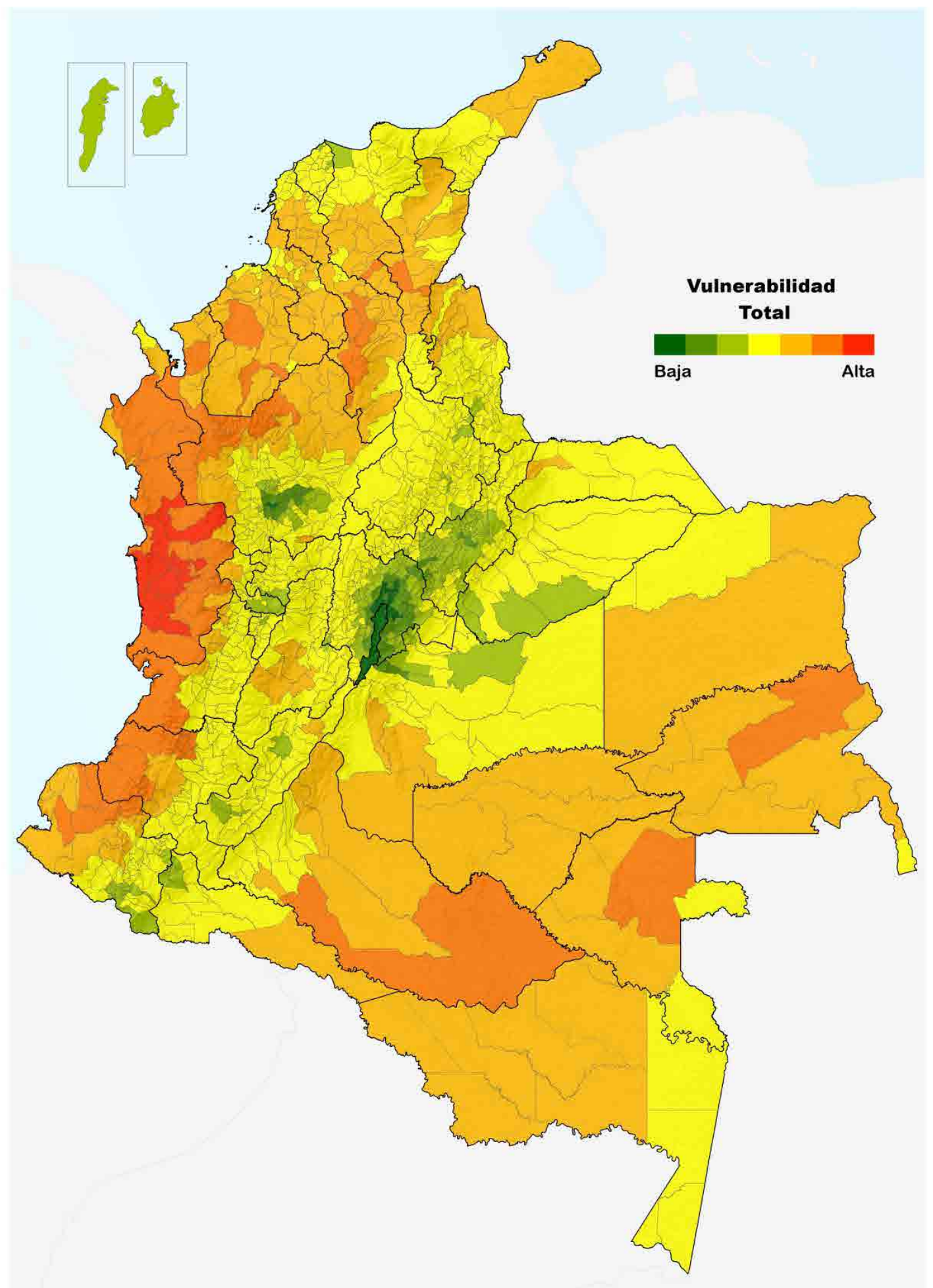

Figura 9. Vulnerabilidad total.

Fuente: Elaboración propia.

Vol. 22 N. ${ }^{\circ} 1$

enero - junio de 2017

pp. 11-32 


\section{Conclusiones}

El análisis espacial permitió determinar las fragilidades del territorio con base en factores seleccionados para cada dimensión de vulnerabilidad. Se establecieron patrones espaciales particulares con comportamientos diferenciados que permiten enfocar a los tomadores de decisiones hacia una intervención efectiva, eficiente y eficaz en aquellas regiones donde la vulnerabilidad es significativa.

Los factores elegidos definen un conjunto de características inherentes a los territorios, que inciden de manera directa o inversa en la vulnerabilidad. Las distribuciones en los datos registraron asimetrías de tipo estadístico, con presencia de valores atípicos, algunos extraordinarios, y otros posibles errores de medición. Se establecieron patrones de concentración, tanto de valores bajos como altos, en todas las variables, confirmados por la estadística espacial global. A nivel local, la mayor incidencia de factores que impactan directamente en la vulnerabilidad social y física se da principalmente en las zonas periféricas del país; en la dimensión económica, el desempeño integral municipal es alto en la región Caribe, mientras que los factores ambientales presentan clústeres de valores bajos en la región Andina.

Los valores más altos de IVS prevalecen en Chocó, y los más bajos, en Cundinamarca. El IVE es alto en Bolívar y Córdoba, y bajo en Antioquia y Cundinamarca. El IVF alto predomina en Santander, y es bajo en Atlántico. El IVA tiene mayor magnitud en Chocó y es menor al interior del país. Los mapas temáticos de vulnerabilidad reflejan las tendencias establecidas de forma individual en los factores explorados, manifestando patrones claros en todas las dimensiones. $\mathrm{Al}$ margen de los límites político-administrativos oficialmente establecidos, la clara delimitación entre la alta y la baja vulnerabilidad constituye una nueva "frontera invisible" definida por las desigualdades existentes entre el desarrollo al interior del país y la marginalidad o exclusión periférica.

Si bien se analizó una cantidad importante de factores, estos no son determinantes absolutos y concluyentes de la vulnerabilidad, ya que esta es una condición espaciotemporal dinámica, por lo que es importante rea- lizar análisis que incorporen características propias de las coyunturas actuales, como el extractivismo minero, el narcotráfico, el cambio climático, la producción agropecuaria y el precio de los alimentos, el ingreso monetario y el mercado laboral, entre otros. Para ello es indispensable que las entidades encargadas generen información detallada y actualizada, de dominio público, con el fin de realizar análisis más complejos que permitan modelar estos fenómenos con el menor grado de generalización e incertidumbre. Así mismo, aunque la vulnerabilidad presenta patrones claros y delimitados sobre el territorio colombiano, esto no implica que al interior de estas zonas este sea el comportamiento general, ya que cada municipio presenta dinámicas particulares internas de exclusión y segregación. De aquí la importancia de establecer diferencias, por ejemplo, entre lo urbano y lo rural, contextos que marcan brechas en las condiciones sociales y ambientales del campo y la ciudad, así como en los entornos espaciales al interior de los conglomerados urbanos, lo que inevitablemente marca procesos de vulnerabilidad a escalas más detalladas que deben ser analizados en cada caso particular.

Indudablemente, la vulnerabilidad adquiere plena importancia y vigencia en la agenda y política pública local, no solo en cuestiones específicas asociadas a la gestión del riesgo, sino desde una perspectiva más amplia, que contempla tanto el desarrollo municipal como temáticas de relevancia actual, entre ellas, la adaptación al cambio climático y el cumplimiento de los Objetivos de Desarrollo Sostenible (ODS). Por lo tanto, la metodología propuesta y los resultados obtenidos se convierten en herramientas e insumos útiles para establecer diagnósticos locales en las distintas dimensiones abordadas o métodos de medición y contraste que permitan evaluar el estado y avance en el cumplimiento de los ODS, que incluyen metas relacionadas con la erradicación de la pobreza, la salud y el bienestar, el agua limpia y el saneamiento, el trabajo decente y el crecimiento económico, la acción por el clima y la vida de ecosistemas, entre otros. Así, este trabajo se convierte en una herramienta útil no solo para los tomadores de decisiones, sino para los analistas, los investigadores y las personas interesadas en estudiar y visualizar la vulnerabilidad como una condición que relaciona sus factores condicionantes y el espacio donde ocurren como un vínculo indisoluble. 


\section{Referencias}

Anselin, L. (1998). Exploratory spatial data analysis in a geocomputational environment. En P. Longley, S. Brooks, R. McDonnell \& B. MacMillan (Eds.), Geocomputation, a primer (pp. 77-94). New York: Wiley.

Aránguez, E., Arribas, M., Aránguez, J. y Ordóñez, J. (2012). Salud y territorio. Aplicaciones prácticas de los sistemas de información geográfica a la salud ambiental. España: Sociedad Española de Sanidad Ambiental. Recuperado de: http://www.madrid.org/cs/Satellite?blobcol= urldata\&blobheader $=$ application\%2Fpdf\&blobheadername $1=$ Content-disposition\&blobheadername2 = cadena\&blobheadervalue $1=$ filename\%3DSESA + optimizado. pdf\&blobheadervalue $2=$ language\%3Des\%26site\%3DPortalSalud\&blobkey=id\&blobtable = MungoBlobs\&blobwhere $=1352807970239 \& s s b i n a r y=$ true .

Armas, I. y Gavris, A. (2013). Social vulnerability assessment using spatial multi-criteria analysis (SEVI model) and the Social Vulnerability Index (SoVI model) - a case study for Bucharest, Romania. Natural Hazards and Earth System Sciences, 13(6), 1481-1499. doi:10.5194/nhess-13-1481-2013.

Bivand, R. S. (2010). Exploratory spatial data analysis. In Handbook of applied spatial analysis (pp. 219-254). Berlín: Springer.

Bortoluzzi, A., Trevignani, V., D’Angelo, C., Mingiaca, M., y Meriggiola, P. (2013). Representación espacial combinada de la vulnerabilidad social y del estado del bosque nativo en el centro norte de la Provincia de Santa Fe, Argentina. Revista digital del Grupo de Estudios sobre Geografía y Análisis Espacial con Sistemas de Información Geográfica (GESIG), 5(5), 96-108. Recuperado de: http://www.gesigproeg.com.ar/documentos/revista-geosig/2013/ARTICULO-05-BORTOLUZZI.pdf.

Burton, C. y Cutter, S. L. (2008). Levee failures and social vulnerability in the Sacramento-San Joaquin Delta area, California. Natural Hazards Review, 9(3), 136149. doi: 10.161/(ASCE)1527-6988(2008)9:3(136).

Busso, G. (2001). Vulnerabilidad social: nociones e implicancias de políticas para Latinoamérica a inicios del siglo XXI. Presentado en el Seminario Internacional "Las diferentes expresiones de la vulnerabilidad social en América Latina y el Caribe", Santiago de Chile, Chile, 20-21 de junio. Recuperado de http://www.cepal.org/ publicaciones/xml/3/8283/GBusso.pdf.

Busso, G. (2005). Pobreza, exclusión y vulnerabilidad social. Usos, limitaciones y potencialidades para el diseño de políticas de desarrollo y de población. Ponencia presentada en las VIII Jornadas Argentinas de Estudios de Población, Tandil, Argentina, 12-14 de octubre. Recuperado de http://www.redaepa.org.ar/jornadas/ viii/AEPA/B10/Busso,\%20Gustavo.pdf.

Castrillón, G. (2009). Análisis espacial de un índice de vulnerabilidad sociodemográfica para los municipios de Cundinamarca. Ponencia presentada en el VIII Seminario 
de Investigación Urbano-Regional ACIUR 2009: Gobierno de municipios y aglomeraciones urbanas, Bogotá, Colombia, 28-30 de septiembre. Recuperado de http://aciur.net/images/documentos/2009/Analisis_Espacial_Indice_VulnerabilidadCastrillon_Gisela-Documento.pdf.

Comisión Económica para América Latina y el Caribe (CEPAL). (8 de octubre de 2002). Capítulos I y V. En Vulnerabilidad sociodemográfica: viejos y nuevos riesgos para comunidades, hogares y personas. LC/W.3. Presentado en el vigésimo período de sesiones de la Comisión en Brasilia, Brasil. Recuperado de http://www.cepal.org/ publicaciones/xml/4/11674/LCW3-Vulnerabilidad.pdf.

Cutter, S. L. (1996). Vulnerability to environmental hazards. Progress in Human Geography, 20(4), 529-539. Recuperado de: http://webra.cas.sc.edu/hvri/docs/ Progress_Human_Geography.pdf.

Cutter, S. L., Boruff, B. J. y Shirley, W. L. (2003). Social vulnerability to environmental hazards. Social Science Quarterly, 84(2), 242-261. Recuperado de: http://webra. cas.sc.edu/hvri/docs/Progress_Human_Geography.pdf.

Cutter, S. L. y Emrich, C. T. (2006). Moral hazard, social catastrophe: The changing face of vulnerability along the hurricane coasts. The Annals of the American Academy of Political and Social Science, 604(1), 102-112. doi: 10.1177/0002716205285515.

De Smith, M. J., Goodchild, M. F. y Longley, P. (2015). Geospatial analysis: a comprehensive guide to principles, techniques and software tools. Recuperado de: http://www.spatialanalysisonline.com/HTML/index.html.

Durán, C. A. (2012). Modelamiento espacial de vulnerabilidad socio-ambiental en los municipios de Colombia. Revista ib Información Básica en Estadística, 2, 43-53.

Dwyer, A., Zoppou, C., Nielsen, O., Day, S. y Roberts, S. (2004). Quantifying social vulnerability: a methodology for identifying those at risk to natural hazards. Recuperado de: http://www.ga.gov.au/webtemp/image_cache/GA4267.pdf.

Fuenzalida, M. (2015). Análisis espacial de las desigualdades territoriales. En M. Fuenzalida, G. Buzai, A. Moreno y A. García de León, Geografía, geotecnología y análisis espacial: tendencias, métodos y aplicaciones (pp. 113-139). Santiago de Chile, Chile: Editorial Triángulo. Recuperado de: http://www.uahurtado.cl/pdf/ Fuenzalida_et_al._2015_Geografa_Geotecnologa_y_Anlisis_Espacial.pdf.

Haining, R. P., Kerry, R. y Oliver, M. (2010). Geography, Spatial Data Analysis, and Geostatistics: And Overview. Geographical Analysis, 42(1), 7-31. Recuperado de: http://www.geog.cam.ac.uk/people/haining/literaturereview.pdf.

Haki, Z. G. (2003). Assessment of social vulnerability using geographic information systems: Pendik, Istanbul case study (tesis de maestría). Middle Eath Technical University, Ankara, Turquía.

Haki, Z., Akyürek, Z. y Düzgün, S. (2004). Assessment of Social Vulnerability Using Geographic Information Systems: Pendik, Istanbul Case Study. Ponencia presentanda en la 7th AGILE Conference on Geographic Information Science, Parallel Session 
4.3 - Environmental/Social Modelling, Heraklion, Grecia, 29 April-1º May. Recuperado de: https://agile-online.org/conference_paper/cds/agile_2004/ papers/4-3-4_haki.pdf.

Huang, J., Su, F., y Zhang, P. (2015). Measuring social vulnerability to natural hazards in Beijing-Tianjin-Hebei Region, China. Chinese Geographical Science, 25(4), 472485. doi: 10.1007/s11769-015-0769-7.

Kienberger, S., Lang, S. y Zeil, P. (2009). Spatial vulnerability units - expert-based spatial modelling of socio-economic vulnerability in the Salzach catchment, Austria. Natural Hazards and Earth System Sciences, 9(3), 767-778. Recuperado de: http://www.nat-hazards-earth-syst-sci.net/9/767/2009/nhess-9-767-2009.pdf.

Lawal, O., y Arokoyu, S. B. (2015). Modelling social vulnerability in sub-Saharan West Africa using a geographical information system. Jàmbá: Journal of Disaster Risk Studies, 7(1), 1-11. Recuperado de: https://www.jamba.org.za/index.php/jamba/ article/viewFile/155/311.

Martin, D. (2005). Geographic Information Systems - Socioeconomic applications. Recuperado de: http://read.pudn.com/downloads161/ebook/725505/ geographic\%20information\%20systems\%20-\%20socioeconomic\%20 applications.pdf.

Nardo, M., Saisana, M., Saltelli, A. y Tarantola, S. (2005). Tools for composite indicators building. 21682 E. European Commission's Joint Research Centre. Recuperado de: http://publications.jrc.ec.europa.eu/repository/bitstream/JRC31473/EUR\%20 21682\%20EN.pdf.

Norman, L. M., Villarreal, M. L., Lara-Valencia, F., Yuan, Y., Nie, W., Wilson, S.,... Sleeter, R. (2012). Mapping socio-environmentally vulnerable populations access and exposure to ecosystem services at the U.S.-Mexico borderlands. Applied Geography, 34, 413-424. doi: 10.1016/j.apgeog.2012.01.006.

Oficina de las Naciones Unidas para la Reducción del Riesgo de Desastres (UNISDR). (2004). Living with risk: a global review of disaster reduction initiatives. Recuperado de: https://www.unisdr.org/files/657_lwr1.pdf.

Olaya, V. (2014). Sistemas de Información Geográfica. Recuperado de: http://www.icog. es/TyT/files/Libro_SIG.pdf.

Tate, E. (2012). Social vulnerability indices: a comparative assessment using uncertainty and sensitivity analysis. Natural Hazards, 63(2), 325-347. doi: 10.1007/s11069012-0152-2.

United States Agency for International Development (USAID). (2014). Design and use of composite indices in assessments of climate vulnerability and resilience. Recuperado de: http://www.ciesin.org/documents/Design_Use_of_Composite_Indices.pdf.

Vergara, R. (2011). Vulnerabilidad social y su distribución espacial: el caso de las entidades federativas de México, 1990-2010. Paradigma económico, 3(2), 85-111. Recuperado de: http://web.uaemex.mx/feconomia/005d.pdf. 
Wang, F., y Wong, D. (2011). Spatial Statistics, Analysis, and Modeling. En C. Yang, D. Wong, Q. Miao y R. Yang (Eds.), Advanced Geoinformation Science. Boca Ratón, Estados Unidos: CRC Press. 